\title{
Manifestações clínicas presentes na doença de Niemann-Pick tipo c em diferentes faixas etárias: uma revisão da literatura
}

\author{
Barbara Beatriz Lira da Silva \\ Acadêmica de Enfermagem da Universidade Estadual do Piauí (UESPI) \\ $\triangle \underline{\text { brbeatriz16@gmail.com }}$ \\ Ana Klara Rodrigues Alves \\ Acadêmica de Enfermagem da Universidade Estadual do Piauí (UESPI) \\ Raquel de Brito Pereira \\ Acadêmica de Enfermagem da Universidade Estadual do Piauí (UESPI) \\ Ana Karla Rodrigues Alve \\ Graduada em Biomedicina pela Universidade Federal do Piauí (UFPI)
}

Kelly Sivocy Sampaio Teixeira

Professora Doutora do curso bacharelado de enfermagem da Universidade Estadual do Piauí (UESPI)

\section{Resumo:}

A doença de Niemann-Pick (DNP) é um distúrbio raro, de armazenamento lisossômico ou de acumulação. É considera uma doença grave e possui três tipos principais: A, B e C. Esse estudo visa analisar se a apresentação de manifestações clínicas em determinada faixa etária do tipo $\mathrm{C}$ da doença influencia na melhora do diagnóstico e no prognóstico do paciente. 0 presente estudo utiliza como método a revisão integrativa da literatura, realizado através de uma busca na Biblioteca Virtual de Saúde (BVS) nas bases de dados do Medline, Pubmed e Scielo (Scientific Electronic Library Online) usando os cruzamentos dos descritores "Doença de Niemann-Pick", "Recém-nascidos". O espectro clínico varia de uma doença pré-natal fatal a uma doença neurodegenerativa crônica no estágio da vida adulta. Os neonatos podem apresentar ascite e hepatomegalia, podendo estar associada à dificuldade respiratória por infiltração dos pulmões. Devido à complexidade da apresentação dos sintomas, o diagnóstico da DNP-C representa um grande desafio, e o reconhecimento dos sintomas e diagnóstico são cruciais para o início precoce de terapias específicas da doença, garantindo melhores resultados aos pacientes. A fim de melhorar o diagnóstico e facilitar a intervenção precoce, foi desenvolvido um ensaio de triagem neonatal com base em biomarcadores plasmáticos de ácidos biliares recentemente identificados. Considerando a prevalência de sintomas no diagnóstico em pacientes de diferentes idades e destacando possíveis correlações entre os sintomas, os profissionais podem obter informações valiosas sobre a sintomatologia da DNP-C, ajudando no reconhecimento de pacientes com DNP-C numa determinada faixa etária.

Palavras-chave: Doença de Niemann-Pick, recém-nascidos, manifestações clínicas, diagnóstico.

\section{Clinical manifestations of Niemann-pick type C disease in different age groups: a literature review}

\begin{abstract}
:
Niemann-Pick disease (NPD) is a rare, lysosomal storage or accumulation disorder. It is considered a serious disease and has three main types: A, B and C. This study aims to analyze whether the presentation of clinical manifestations in a certain age group of type $C$ of the disease influences the
\end{abstract}


improvement of the diagnosis and the patient's prognosis. This study uses the integrative literature review as a method, carried out by searching the Virtual Health Library (VHL) in the Medline, Pubmed and Scielo (Scientific Electronic Library Online) databases using the intersections of the descriptors "Disease Niemann-Pick", "Newborns". The clinical spectrum varies from a fatal prenatal disease to a chronic neurodegenerative disease in the adult stage. Neonates may have ascites and hepatomegaly, and may be associated with breathing difficulty due to infiltration of the lungs. Due to the complexity of the presentation of symptoms, the diagnosis of NPC represents a great challenge, and the recognition of symptoms and diagnosis are crucial for the early initiation of specific therapies of the disease, ensuring better results for patients. In order to improve diagnosis and facilitate early intervention, a neonatal screening assay based on recently identified plasma bile acid biomarkers has been developed. Considering the prevalence of symptoms in the diagnosis in patients of different ages and highlighting possible correlations between symptoms, professionals can obtain valuable information about the symptoms of NPC, helping in the recognition of patients with NPC in a certain age group.

Keywords: Niemann-Pick disease, newborns, clinical manifestations, diagnosis.

\section{Manifestaciones clínicas presentes en la enfermedad de Niemann-pick tipo $C$ en diferentes grupos de edad: una revisión de la literatura}

\section{Resumen:}

La enfermedad de Niemann-Pick (DNP) es un trastorno raro de almacenamiento de lisosomas o de acumulación. Es considerada una enfermedad grave y posee tres tipos principales: A, B y C. Este estudio tiene el objetivo de analizar si la presentación de manifestaciones clínicas en un determinado grupo de edad del tipo $\mathrm{C}$ de la enfermedad influye en la mejora del diagnóstico y en el pronóstico del paciente. El presente estudio utiliza como método la revisión integrativa del marco teórico, realizado a través de la búsqueda en la Biblioteca Virtual de Salud (BVS) en las bases de datos Medline, Pubmed y Scielo (Scientific Electronic Library Online) utilizando las intersecciones de los descriptores "Enfermedad de Niemann-Pick, "Recién nacidos". El espectro clínico cambia desde una enfermedad prenatal mortal hasta una enfermedad neurodegenerativa crónica en la etapa adulta. Los recién nacidos pueden presentar ascitis y hepatomegalia, y puede estar asociada con la dificultad respiratoria debido a la infiltración de los pulmones. Debido a la complejidad de la presentación de los síntomas, el diagnóstico de la DNP-C representa un gran desafío, y el reconocimiento de los síntomas y del diagnóstico son cruciales para el inicio precoz de terapias específicas de la enfermedad, asegurando mejores resultados a los pacientes. Con la intención de mejorar el diagnóstico y facilitar la intervención precoz, fue desarrollado un ensayo de detección neonatal basado en biomarcadores de ácido biliar en plasma recientemente identificados. Teniendo en cuenta la prevalencia de síntomas en el diagnóstico en pacientes de diferentes edades y destacando posibles correlaciones entre los síntomas, los profesionales pueden obtener informaciones valiosas sobre la sintomatología de la DNP$C$, ayudando en el reconocimiento de pacientes con DNP-C en un determinado grupo de edad.

Palabras clave: Enfermedad de Niemann-Pick, recién nacidos, manifestaciones clínicas, diagnóstico.

\section{INTRODUÇÃO}

A doença de Niemann-Pick (DNP) é um distúrbio raro, de armazenamento lisossômico ou de acumulação, cuja prevalência de nascimento é estimada em 0,4-0,6/ 100.000. A deficiência da enzima lisossômica esfingomielinase ácida resulta no acúmulo progressivo de esfingomielina nos hepatócitos, nas células reticuloendoteliais e, em casos graves, nos neurônios (CASSIMAN et al., 2016). Os sintomas são devidos ao acúmulo de macrófagos cheios 
de lipídios vacuolados, conhecidos como células Niemann-Pick, em vários órgãos, como fígado, baço, medula óssea, pulmão e sistema nervoso central (FREITAS et al., 2017).

É considera uma doença generalizada e grave. Possui três tipos principais, o tipo A e o tipo B são distúrbios de armazenamento lisossômico autossômico recessivo causados por mutações homozigóticas ou heterozigotas compostas no gene da esfingomielina fosfodiesterase 1, levam à produção e função deficientes da enzima esfingomielinase ácida lisossômica. A DNP tipo A é a forma severa de início infantil, associada ao envolvimento visceral e à neurodegeneração progressiva, que na maioria das vezes leva à falha no crescimento e morte aos 3 anos de idade e a DNP tipo B, é uma forma não neurológica de início tardio caracterizada por hepatoeslenomegalia, que geralmente é compatível com a sobrevivência de um adulto (RANGANATH et al., 2016; CASSIMAN et al., 2016).

O tipo C (DNP-C) é um distúrbio neurodegenerativo raro de armazenamento de colesterol causado por mutações nos genes NPC1 ou NPC2. As proteínas que codificam esses genes estão envolvidas na exportação adequada de colesterol e é possível que ajam em conjunto para regular o transporte lipídico intracelular e a homeostase (ECHEVERRIA et al., 2017). A DNP-C é caracterizada por uma ampla gama de sintomas viscerais, neurológicos e psiquiátricos que podem variar consideravelmente entre os pacientes. A anormalidade funcional causa acúmulo de colesterol nos lisossomos e, posteriormente, nos endossomos (KUMAGAI et al., 2019). Sua prevalência é de 1:120.000-150.000 nascidos vivos (STARETZCHACHAM et al., 2018).

A apresentação clínica da doença é variável e a idade de início varia desde a idade perinatal período até a idade adulta. A doença é tipicamente caracterizada por sintomas viscerais e neurológicos. Além de um pequeno grupo de pacientes apresentando uma forma perinatal grave levando à morte dentro do primeiro ano de vida devido a insuficiência hepática ou respiratória, a maioria dos pacientes desenvolve uma progressiva doença neurológica. De fato, o DNP-C tem sido tradicionalmente classificado com base na idade de início da os sintomas neurológicos, independentemente da idade do primeiro sintoma (DARDIS et al., 2020).

Pode ocorrer desde o período perinatal até a idade adulta. É classificada em: forma infantil grave (início aos 2 anos), forma infantil tardia (início aos 3-5 anos), forma juvenil 
(início aos 5-16 anos) ou forma adulta (início aos > 16 anos). 0 prognóstico é variável, com a forma mais grave ocorrendo nos dois primeiros anos de vida. (SANTED et al., 2017).

O presente estudo tem como finalidade verificar se a apresentação de manifestações clínicas em determinada faixa etária da doença de Niemann-Pick tipo C influencia a melhora do diagnóstico tardio e melhora no prognostico do paciente.

\section{METODOLOGIA}

O presente estudo utiliza como método a revisão integrativa da literatura, realizado através de uma busca na Biblioteca Virtual de Saúde (BVS) nas bases de dados do Medline, Pubmed e Scielo (Scientific Electronic Library Online) usando os cruzamentos dos descritores "Doença de Niemann-Pick", "Recém-nascidos". Para a construção deste artigo, aplicou-se o seguinte questionamento: A apresentação de manifestações clínicas de acordo com a faixa etária da doença de Niemann-Pick tipo $\mathrm{C}$ antecipa o diagnóstico e melhora a sobrevida dos pacientes?

Quanto aos critérios de inclusão, introduziram-se artigos escritos nas línguas espanhola e inglesa; publicados entre os anos 2015 a 2020 e que retrataram a sintomatologia, diagnóstico e tratamento da DNP-C. No que diz respeito aos critérios de exclusão, dispensaram-se artigos que se distanciavam da temática central desta revisão e trabalhos que não apresentassem resumos na íntegra nas bases de dados pesquisadas. Após a pré-leitura e leitura seletiva dos textos, foram selecionados 22 artigos (Quadro 1), nos quais realizou-se uma leitura interpretativa buscando responder à pergunta de pesquisa desta revisão.

Foi realizada uma análise ampla da literatura com passos pré-definidos, buscando responder o questionamento, descritores, e seus critérios de inclusão e exclusão. Foram encontrados 75 artigos, sendo 22 selecionados, destes 5 foram encontrados na Scielo, 9 na base de dados Pubmed e o restante encontrado na base de dados Medline, somado a esses foi utilizado o manual do Ministério da Saúde. 
Quadro 1 - Distribuição dos artigos utilizados nesta revisão segundo o nome da publicação, autores, revista e ano.

\begin{tabular}{|l|l|l|l|}
\hline \multicolumn{1}{|c|}{ ARTIGOS } & \multicolumn{1}{|c|}{ AUTORES } & ANO DE \\
PUBLICAÇÃo
\end{tabular}




A case of Niemann-Pick
disease type C with neonatal
liver failure initially
diagnosed as neonatal
hemochromatosis.

Information and Diagnosis Networks - tools to improve diagnosis and treatment for patients with rare genetic diseases.

Diretrizes Brasileiras para Diagnóstico e Tratamento da Doença de Niemann-Pick tipo C.

Newborn presentation of Niemann-Pick disease type $C$ - Difficulties and limitations of diagnostic methods.

Pulmonary involvement in Niemann-Pick C type 1

Niemann-Pick disease type B: HRCT assessment of pulmonary involvement.
Tadayuki Kumagai, Hiroshi Terashima, Hajime Ushida, Aya Narita, Yoshikatsu Eto, Masaya Brain Dev 2019 Kubota.

Arun Babu Kumar, Xinying Hong, Fan Yi, Tim Wood, Michael $\mathrm{H}$. Gelb.

Molecular

Genetics

and

2019

Metabolism

Helena Moreira Silva, Inês Maio, Anabela Bandeira, Esmeralda Gomes Martins, Ermelinda Santos Silva.

European Journal of 2019 Pediatrics

Genetics

and

Molecular

Doederlein Schwartz, Mariluce Riegel, Carmen Regla Vargas, Maira Graeff Burin, Sandra Leistner-Segal, Patrícia AshtonProlla, and Roberto Giuglian.

Biology

Patryk Lipinski, Irena Jankowska, Agnieszka Ługowska, Małgorzata Pediatr Musielak, Maciej Pronicki, Anna Neonatol Tylki-Szymanska.

Ornan Staretz-Chacham, M. European Aviram, I. Morag, U. Goldbart, E. Journal of 2018 Hershkovitz. Pediatrics

Heloisa Maria Pereira Freitas, Alexandre Dias Mançano, Rosana Souza Rodrigues, Bruno Hochhegger, Pedro Paulo Teixeira J. bras. pneumol 


\begin{tabular}{|c|}
\hline $\begin{array}{l}\text { Niemann-Pick disease type } C \\
\text { in the newborn period: a } \\
\text { single-center experience. }\end{array}$ \\
\hline $\begin{array}{l}\text { Differences in Niemann-Pick } \\
\text { disease Type C } \\
\text { symptomatology observed in } \\
\text { patients of different ages. }\end{array}$ \\
\hline $\begin{array}{l}\text { Enfermedad de Niemann } \\
\text { Pick tipo-A. Presentación de } \\
12 \text { casos. }\end{array}$ \\
\hline $\begin{array}{l}\text { Ciclodextrina intratecal en el } \\
\text { tratamiento de la } \\
\text { enfermedad de } \\
\text { Pick tipo C }\end{array}$ \\
\hline $\begin{array}{l}\text { Cause of death in patients } \\
\text { with chronic visceral and } \\
\text { chronic neurovisceral acid } \\
\text { sphingomyelinase deficiency } \\
\text { (Niemann-Pick disease type } \\
\text { B and B variant): Literature } \\
\text { review and report of new } \\
\text { cases }\end{array}$ \\
\hline $\begin{array}{l}\text { Visceral symptoms as a key } \\
\text { diagnostic sign for the early } \\
\text { infantile form of Niemann- } \\
\text { Pick disease type C in a } \\
\text { Russian patient: a case } \\
\text { report. }\end{array}$ \\
\hline $\begin{array}{l}\text { Diagnóstico bioquímico } \\
\text { positivo en pacientes con } \\
\text { sospecha clínica de Niemann } \\
\text { Pick C }\end{array}$ \\
\hline $\begin{array}{l}\text { A Suspicion Index to aid } \\
\text { screening of early-onset } \\
\text { Niemann-Pick disease Type C } \\
(\mathrm{NP}-\mathrm{C}) \text {. }\end{array}$ \\
\hline
\end{tabular}

Cesar Augusto Araujo Neto, Edson Marchiori.

Ersin Gumus, Goknur Haliloglu, Asuman Nur Karhan, Hulya Demir, Figen Gurakan, Meral Topcu, Aysel Yuce.

Eugen Mengel, MercedesPineda, Christian J.Hendriksz, Mol Genet MarkWalterfang, Juan V.Torres, Metab

European Journal of 2017 Pediatrics Stefan A. Kolb.

J Zarco-Román, HE RomeroGómez, L Carbajal-Rodríguez

Acta pediátrica de México

María Roch Santed, María Josep Cabañas Poy, Carme Cañete Ramírez, Aurora Fernández Polo, Mireia Del Toro Riera, Farmacia Hospitalaria Susana Clemente Bautista.

David Cassiman, Seymour Packman, Bruno Bembi,

Hadhami Ben Turkia, Moeenaldeen Al-Sayed, Manuel Mol. Genet. Schiff, Jackie Imrie, Paulina Mabe, Tsutomu Takahashi, Karl Eugen Mengel, Roberto Giugliani, Gerald F.Cox.

A. V. Degtyareva, S. V. Mikhailova, E. Y. Zakharova, E. L. Tumanova, A. A. Puchkova.

J Med Case Rep.

Liz Carolina Pardo Echeverría, Leopoldo Antonio Arrieta Violet, Fernanda Timm Seabra Souza.

Ciencia en Desarrollo

Mercedes Pineda, Eugen Mengel, Helena Jahnová, Bénédicte Héron, BMC Jackie Imrie, Charles M. Lourenço, Pediatr 2016 Vanessa van der Linden, Parvaneh 


\begin{tabular}{|c|c|c|c|}
\hline & $\begin{array}{l}\text { Karimzadeh, } \quad \text { Vassili } \\
\text { Valayannopoulo, Pavel Jesina, } \\
\text { Juan V. Torres, Stefan A. Kolb. }\end{array}$ & & \\
\hline $\begin{array}{l}\text { Spectrum of } \\
\text { Mutations in Asian-Indian } \\
\text { Patients with Acid } \\
\text { Sphingomyelinase (ASM) - } \\
\text { Deficient Niemann-Pick } \\
\text { Disease }\end{array}$ & $\begin{array}{l}\text { Prajnya Ranganath, Divya Matta, } \\
\text { Gandham SriLakshmi Bhavani, } \\
\text { Savita Wangnekar, Jamal } \\
\text { Mohammed Nurul Jain, Ishwar C. } \\
\text { Verma, Madhulika Kabra, Ratna } \\
\text { Dua Puri, Sumita Danda, Neerja } \\
\text { Gupta, Katta M. Girisha, Vaikom } \\
\text { H. Sankar, Siddaramappa J. Patil, } \\
\text { Akella Radha Ramadevi, } \\
\text { Meenakshi Bhat, Kalpana } \\
\text { Gowrishankar, Kausik Mandal, } \\
\text { Shagun Aggarwal, Parag Mohan } \\
\text { Tamhankar, Preetha Tilak, } \\
\text { Shubha R. Phadke, and Ashwin } \\
\text { Dalal. }\end{array}$ & $\begin{array}{ll}\text { Am J } & \text { Med } \\
\text { Genet } & \text { Part } \\
\text { A } & \end{array}$ & 2016 \\
\hline
\end{tabular}

Fonte: própria.

\section{RESULTADOS}

O espectro clínico varia de uma doença pré-natal fatal a uma doença neurodegenerativa crônica no estágio da vida adulta. Os neonatos podem apresentar ascite e hepatomegalia, podendo estar associada à dificuldade respiratória por infiltração dos pulmões. Outros lactentes, sem doença hepática ou pulmonar, podem ter hipotonia e atraso no desenvolvimento, ou seja, podem ter danos neurológicos (BRASIL, 2019).

Com relação às manifestações clínicas, as mesmas são características de acordo com a faixa etária, foi verificado que sintomas viscerais, como esplenomegalia e icterícia em pacientes com idade $\leq 4$ anos, enquanto os sintomas neurológicos e psiquiátricos são predominante em crianças e adolescentes mais velhos (4-16 anos) e adultos maiores de 16 anos. Os sintomas neurológicos incluem paralisia supranuclear vertical (VSGP) do olhar, distonia, demência, ataxia, disfagia, convulsões, todas essas manifestações observadas em pacientes com idade acima de 4 anos, em alguns casos pode ocorrer morte na segunda ou terceira década de vida por pneumonia aspirativa (MENGEL et al., 2017 e CACHAM et al., 2018). 
As manifestações clínicas são diversas e, por vezes, os pacientes podem apresentar sintomas inespecíficos, apresentam variações com a idade do período antenatal à idade adulta (até sétima década de vida). As manifestações clínicas mais comumente associadas à doença de NPC são paralisia do olhar supranuclear vertical, cataplexia gelástica, esplenomegalia isolada, icterícia ou colestase neonatal prolongada, demência ou disfunção cognitiva precoce. A sobrevida dos pacientes varia de alguns dias a mais de 60 anos de idade, embora na maioria dos casos o óbito ocorra entre 10 e 25 anos de idade. A doença de NPC pode ser classificada de acordo com a idade de início das manifestações clínicas em 5 grupos distintos: perinatal, infantil precoce, infantil tardio, juvenil, adolescente/ adulto. (ECHEVERRÍA et al., 2016; DEGTYAREVA et al., 2019).

Com relação ao período perinatal início dos sintomas antes de 2 meses de idade, as manifestações são sistêmicas ocorre: hepatomegalia, esplenomegalia, colestase neonatal, plaquetopenia, insuficiência hepática, hidropsia fetal, ascite fetal, restrição de crescimento intrauterino e doença pulmonar, somada a manifestações neurológicas como hipotonia, pode surgir icterícia que desaparece na maioria dos casos de forma espontânea já a visceromegalia persiste por tempo variável. Os sintomas neurológicos são tardios. Em cerca de 8-9\% dos casos a doença pode progredir rapidamente para insuficiência hepática e/ou falência de múltiplos órgãos e óbito em 6 meses. Em alguns casos a apresentação inicial pode ser ascite/hidropsia fetal. Na fase infantil precoce início entre 2 meses e 2 anos de idade, apresentam as mesmas manifestações sistêmicas, podendo ocorrer hepatoesplenomegalia ou esplenomegalia (isolada ou com manifestações neurológicas) e colestase prolongada, com relação aos danos neurológicos podem acontecer: hipotonia central, retardo do desenvolvimento motor, atraso da fala, disfagia, espasticidade, paralisia do olhar vertical supranuclear (POVSN), apresentando a presença de hipotonia e atraso do desenvolvimento motor. A hepatoesplenomegalia e/ou icterícia neonatal prolongada quase sempre são observadas (ECHEVERRÍA et al., 2016; SANTED et al., 2017)

Infantil tardio: início entre 2 e 6 anos de idade, sintomatologia semelhante as da infantil preoce, podendo ser acrescentado a história de icterícia neonatal colestática prolongada, manifestações neurológicas: retardo/regressão do desenvolvimento, atraso da fala, dificuldades de controle motor, quedas frequentes, ataxia progressiva, distonia, disartria, disfagia, convulsões, cataplexia, POVSN e déficit auditivo. O paciente apresenta 
dificuldades de controle motor e distúrbio da marcha. Podem ser notados atraso da fala, história de colestase neonatal e visceromegalia variável. POVSN está tipicamente presente, porém muitas vezes não é reconhecida. O sintoma inicial pode ser a surdez sensorial, podendo ocorrer perda auditiva em altas frequências, anormalidades nos reflexos acústicos ou nas respostas do tronco cerebral no que diz respeito à audição, sugerindo alterações generalizadas ou a cataplexia gelástica, representada pela perda repentina de tônus muscular resultando em quedas ou colapso, A epilepsia subsequente é muito frequente. Na fase juvenil: - Início entre 6 e 15 anos de idade, somado as alterações em fígado e baço, as manifestações neurológicas/psiquiátricas podem representar um agravo muito grande nos doentes como: baixo rendimento escolar, dificuldade de aprendizado, perda da habilidade de linguagem, quedas frequentes, dificuldades de controle motor, ataxia progressiva, distonia, dismetria, disartria, disfagia, POVSN, cataplexia gelástica, convulsões, distúrbios de comportamento. A forma juvenil é a segunda forma mais frequente de apresentação de NPC e se manifesta como distúrbio cognitivo, problemas de coordenação e POVSN. Adolescente/adulto: - Início após 15 anos de idade: esplenomegalia, alterações neurológicas/psiquiátricas declínio cognitivo, demência, dificuldade de aprendizado, esquizofrenia, depressão, dificuldades de controle motor, sintomas motores progressivos, tremor, ataxia, distonia/discinesia, disartria, disfagia, POVSN, a forma inicial em adolescentes e adultos corresponde a até um terço dos casos de doença de NPC. Prejuízo cognitivo ocorre invariavelmente e tem maior taxa de manifestações neurológicas e psiquiátricas. $\mathrm{O}$ atraso diagnóstico é comum, sendo minimizada se POVSN for identificada (SANTED et al., 2017; RODRÍGUEZ et al., 2019).

\section{DISCUSSÃo}

Devido à complexidade da apresentação dos sintomas, o diagnóstico de DNP-C apresenta um grande desafio na prática clínica, mas o reconhecimento oportuno dos sintomas e o diagnóstico de NP-C são cruciais para apoiar o início precoce de terapias sintomáticas e específicas da doença e garantir os melhores resultados possíveis para os pacientes (MENGEL et al., 2017). 
No estudo de Jiang (2019) para melhorar o diagnóstico e facilitar a intervenção precoce, foi desenvolvido um ensaio de triagem neonatal combase em biomarcadores plasmáticos de ácidos biliares recentemente identificados .Como a triagem neonatal foi validada usando manchas de sangue seco(DBS) de pacientes com NPC1 já diagnosticados, uma pergunta sem resposta era se a triagem seria capaz de detectar indivíduos com NPC1 no nascimento. Implementação de ensaios de diagnóstico baseados em biomarcadores sensíveis e específicos baseados em sangue, incluindo colestano$3 \beta, 5 \alpha, 6 \beta$-triol, seus metabólitos do ácido biliar lysoSM509 contribuíram para um diagnóstico mais oportuno de DNP-C, mas não elimina o atraso diagnóstico.

Geralmente, as elevações da atividade de biomarcadores são mais distintas em pacientes com DNP-C com início clínico precoce do que em pacientes com início tardio. Foi relatado que os oxisteróis plasmáticos, por exemplo, colestano-3 $\beta, 5 \alpha$ e $6 \beta$-triol, são nitidamente elevados na maioria, mas não em todos os pacientes com DNP-C, e elevações também podem ser encontradas em outras condições de doença. O teste genético, como regra, é obrigatório em qualquer caso suspeito de NP-C. (JECEL et al., 2019). O oxysterol é um marcador de triagem útil para o diagnóstico de NPC no período neonatal, pode servir como uma abordagem de triagem útil para identificar novos casos de NP-C e pode ajudar a detectar candidatos a testes genéticos moleculares (DEGTYAREVA et al., 2019; KUMAGAI et al., 2019).

O diagnóstico bioquímico requer a cultura de fibroblastos para detectar a deposição de colesterol não colesterol esterificados em vesículas perinucleares, considera-se que 8085\% dos pacientes afetados têm intensa fluorescência (ECHEVERRÍA et al., 2016).

A raridade da doença e a escassez de competência levam ao erro ou atraso de diagnóstico e impedimento ao cuidado adequado, com desgaste emocional dos pacientes e de suas famílias, assim como das equipes de saúde. O encaminhamento para teste diagnóstico em NP-C depende do reconhecimento de os sinais e sintomas de NP-C. No entanto, a detecção pode ser difícil devido à apresentação clínica extremamente heterogênea e geralmente os profissionais médicos tem baixo conhecimento sobre a patologia. As variáveis, início da doença e amplas formas de manifestações clínicas dificultam e retardam o diagnóstico e desse modo contribuem para sub-detecção de NP-C e, em alguns casos, seu diagnóstico incorreto. É de total conhecimento que um diagnóstico exato e imediato é de total eficiência para um encaminhamento apropriado do quadro clínico, proporciona melhoria da qualidade de vida. A proposta é criar uma estratégia da abordagem terapêutica de suporte sintomático, provida 
por equipe multiprofissional e interdisciplinar (MENGEL et al., 2017; GUMUS et al., 2017, BRASIL, 2019).

Não há ainda terapia curativa doença-específica disponível para NPC, e a doença progride geralmente para morte prematura. A terapia é baseada no tratamento sintomático e na administração do medicamento Miglustat, que é um inibidor da glicosilceramida sintase, inibindo, portanto, a síntese de glicolipídios podendo retardar a progressão de manifestações neurológicas irreversíveis e é o único tratamento aprovado para a doença. Também reduz o acúmulo de gangliosídeos GM2 e GM3, glicosilceramida e lactosilceramida. (SANTED et al., 2017; PINEDA et al., 2016).

\section{CONCLUSÃO}

Considerando a prevalência de sintomas no diagnóstico em pacientes de diferentes idades e destacando possíveis correlações entre os sintomas, os profissionais podem obter informações valiosas sobre a sintomatologia do DNP-C. Esta informação pode ajudar no reconhecimento de pacientes com DNP-C dentro de uma determinada faixa etária. Essas análises fornecem uma revisão lógica e oportuna da sintomatologia característica dos pacientes diagnosticados com NP-C em todas as idades e contribuem para a nossa compreensão da doença, seu início e progressão variáveis.

O diagnóstico bioquímico positivo associado à DNP-C, através da intensidade da fluorescência perinuclear, facilitou a visualização do depósito lisossômico. No entanto, o diagnóstico definitivo de deve ser complementado por estudos moleculares para mutações nos genes DNPC-1 e DNPC-2. Além disso, esses dados devem ser correlacionados com as manifestações clínicas dos pacientes avaliados para evitar subdiagnóstico, falsos positivos e má interpretação com diagnósticos diferenciais que também mostram forte fluorescência, mas suas manifestações clínicas não estão associadas ao NPC.

No Brasil, para melhorar o acesso ao seu diagnóstico, a Rede NP-C Brasil foi criada em 2009 com o objetivo de fornecer a médicos de diversas especialidades triagem e testes de diagnóstico para identificar pacientes com NP-C no Brasil e em outros países. A Rede NPC 
Brasil pode ser acessada por qualquer serviço que avalie pacientes que possam apresentar NP-C.

\section{REFERÊNCIAS}

BRASIL. Diretrizes Brasileiras para Diagnóstico e Tratamento da Doença de Niemann-Pick tipo C. Ministério da Saúde. Secretaria de Ciência, Tecnologia e Insumos Estratégicos, Comissão Nacional de Incorporação de Tecnologias no Sistema Único de Saúde (Conitec) - Brasília, 2019.

CASSIMAN, D.; PACKMANB, S.; BEMBIC, B.; TURKIA, H. B.; AL-SAYED, M.; SCHIFF, M.; IMRIEJ.; MABE, P.; TAKAHASHI, T.; MENGEL, K. E.; GIUGLIANI, R.; COX, G. F.. Cause of death in patients with chronic visceral and chronic neurovisceral acid sphingomyelinase deficiency (Niemann-Pick disease type B and B variant): Literature review and report of new cases. Mol. Genet. Metab. v. 118, p. 206-213, 2016.

CHACHAM, O. S.; AVIRAM, M.; MORAG, I.; GOLDBART, U.; HERSHKOVITZ;E.. Pulmonary involvement in Niemann-Pick C type 1. European Journal of Pediatrics. v.177, n.11, p. 1609-1615, Nov. 2018.

DARDIS, A.; ZAMPIERI, S.; GELLERA, C.; CARROZZO, R.; CATTAROSSI, S.; PERUZZO, P.; DARIOL, R.; SECHI, A.; DEODATO, F.; CACCIA, C.; VERRIGNI, D.; GASPERINI, S.; FIUMARA, A.; FECAROTTA, S.; CARECCHIO, M.; FILOSTO, M.; SANTORO, L.; BORRONI, B.; BORDUGO, A.; BRANCATI, F.; RUSSO, C. V.; ROCCO, M.D.; SCARPA, A. T. M., BEMBI, B. Molecular Genetics of NiemannPick Type C Disease $\quad$ in Italy: An Update on 105 Patients and Description of 18 NPC1 Novel Variants. J. Clin. Med., v.9, n.679, 2020.

DEgTYAREVA, A. V.; MIKHAIlOVA, S. V.; ZAKHAROVA E. Y.; TUMANOVA, E. L.; PUCHKOVA, A. A.. Visceral symptoms as a key diagnostic sign for the early infantile form of Niemann-Pick disease type $C$ in a Russian patient: a case report. J Med Case Rep. v. 10, n. 1, p. 143, 2016.

DEGTYAREVA, A. V.; PROSHLYAKOVA, T. Y.; GAUTIER, M. S.; DEGTYAREV, D. N.; KAMENETS, E. A.; BAYDAKOVA, G. V.; REBRIKOV, D. V.; ZAKHAROVA, E. Y.. Oxysterol/chitotriosidase based selective screening for NiemannPick type $C$ in infantile cholestasis syndrome patients. BMC Med Genet, v. 20, n. 1, p.123, 2019.

ECHEVERRIA, L. P. C.; VIOLET, L. A. A.; SOUZA, F. S. T.. Diagnóstico bioquímico positivo en pacientes con sospecha clínica de Niemann Pick C. Ciencia en Desarrollo, v. 7, n. 2, p.31 - 41, 2016.

FREITAS, H. M. P.; MANÇANO, A. D.; RODRIGUES, R. S.; HOCHHEGGER, B.; TORRES, P. P. T. S.; ESCUISSATO, D.; NETO, C. A. A.; MARCHIORI, E.. Niemann-Pick disease type B: HRCT assessment of pulmonary involvement. J. bras. pneumol. vol.43 n.6, São Paulo nov./ dez. 2017.

GABANDÉ-RODRÍGUEZ, E.; PÉREZ-CAÑAMÁS, A.; SOTO-HUELIN, B.; MITROI, D. N.; SÁNCHEZ-REDONDO, S.; MARTÍNEZ-SÁEZ, E.; VENERO, C.; PEINADO, H.; LEDESMA, M. D.. Lipid-induced lysosomal damage after demyelination corrupts microglia protective function in lysosomal storage disorders. EMBO J, v. 38, n. 2, p. 0115, 2019.

GUMUS, E.; HALILOGLU, G.; KARHAN, A. N.; DEMIR, H.; GURAKAN, F.; TOPCU, M.; YUCE, A..Niemann-Pick disease type $C$ in the newborn period: a single-center experience. Eur J Pediatr, v. 176, p. 1669-1676, 2017.

JECEL, J.; HARZER, K.; PASCHKE, E.; BECK-WÖDL, S.; BAUER, P.; HEJTMAN, M.; KATZENSCHLAGER, R.. Distinct Niemann-Pick Disease Type C Clinical, Cytological, and Biochemical Phenotype in an Adult Patient With 1 Mutated, Overexpressed NPC1 Allele. J. erros inatos metab. t. Porto Alegre, v. 3, 2019. 
JIANG, X.; SIDHU, R.; ORSINI, J. J.; FARHAT, N. Y.; PORTER, F. D.; BERRY-KRAVIS, E.; SCHAFFER, J. E.; ORY, D. S.. Diagnosis of niemann-pick C1 by measurement of bile acid biomarkers in archived newborn dried blood spots. Mol Genet Metab, v. 126, n. 2, p. 183-187, 2019.

KUMAGAI, T.; TERASHIMA, H.; UCHIDA, H.; NARITA, A.; ETO, Y.; KUBOTA, M.. A case of Niemann-Pick disease type $C$ with neonatal liver failure initially diagnosed as neonatal hemochromatosis. Brain Dev, v. 41, n. 5, p. 460464, 2019.

KUMAR, A.B., HONGA, X., YI, F., WOOD, T., GELB, M. H. Tandem mass spectrometry-based multiplex assays for $\alpha$-mannosidosis and fucosidosis. Molecular Genetics and Metabolism, v.127, p.207-211, 2019.

LIPINSKI, P.; JANKOWSKA, I.; LUGOWSKA, A.; MUSIELAK, M.; PRONICKI, M.; TYLKI-SZYMANSKA, A.. Newborn presentation of Niemann-Pick disease type C - Difficulties and limitations of diagnostic methods. Pediatr Neonatol, v. 59, n. 3, p. 317-318, 2018.

MENGEL, E.; PINEDA, M.; HENDRIKSZ, C. J.; WALTERFANG, M.; TORRES, J. V.; KOLB, S. A.. Differences in NiemannPick disease Type C symptomatology observed in patients of different ages. Mol Genet Metab, v. 120, n. 3, p. 180-189, 2017.

PINEDA, M.; MENGEL, E.; JAHNOVÁ, H.; HÉRON,B.; IMRIE, J.; LOURENÇO,C. M.; LINDEN, V. V.; KARIMZADEH, P., VALAYANNOPOULO, V.; JESINA, P.; TORRES, J. V.; KOLB, S. A.. A Suspicion Index to aid screening of early-onset Niemann-Pick disease Type C (NP-C). BMC Pediatr v. 16, n. 107, 2016.

RANGANATH, P.; MATTA, D.; BHAVANI, G. S.; WANGNEKAR,S.; JAIN, J. M. N.; VERMA, I. C.; KABRA, M.; PURI, R. D.; DANDA, S.; GUPTA, N.; GIRISHA, K .M.; SANKAR, V. H.; PATIL, S. J.; RAMADEVI, A. R.; BHAT, M.; GOWRISHANKAR, K.; MANDAL, K.; AGGARWAL, S.; TAMHANKAR, P. M.; TILAK, P.; PHADKE, S.R.; DALAL, A.. Spectrum of SMPD1 Mutations in Asian-Indian Patients with Acid Sphingomyelinase (ASM)-Deficient NiemannPick Disease. Am J Med Genet Part A, v.170, p.2719-2730.

ROMÁN, J. Z:; GÓMEZ, R.; RODRÍGUEZ, L. C.. Enfermedad de Niemann Pick tipo-A. Presentación de 12 casos. Acta pediátrica de México, v. 38, n. 3, p.152 - 164, 2017.

SANTED, M. R.; POY, M. J. C.; RAMÍREZ, C. C.; POLO, A.F.; RIERA, M.T.; BAUTISTA, S.C. Ciclodextrina intratecal en el tratamiento de la enfermedad de Niemann Pick tipo C. Farmacia Hospitalaria Jun, v. 41, n. 3, p. 435 - 438, 2017.

SILVA, H. M.,MAIO, I., BANDEIRA, A., MARTINS, E. G., SILVA, E. S. Metabolic liver diseases presenting with neonatal cholestasis: at the crossroad between old and new paradigms. European Journal of Pediatrics, Jan. 2019 .

STARETZ-CHACHAM, O., AVIRAM, M., MORAG, I., GOLDBART, U., HERSHKOVITZ, E. Pulmonary involvement in Niemann-Pick C type 1. Eur J Pediatr, v. 177, n. 11, p. 1609-1615, 2018.

VIEIRA, T. A., TRAPP, F. B., SOUZA, C. F. M., FACCINI, L. S., JARDIM, L. B., SCHWARTZ, I. V. D., RIEGEL, M., VARGAS, C. R., BURIN, M. G. LEISTNER-SEGAL, S., ASHTON-PROLLA, P., GIUGLIAN, R. Information and Diagnosis Networks - tools to improve diagnosis and treatment for patients with rare genetic diseases. Genetics and Molecular Biology, v. 42, n. 1, p. 155 - 164, 2019.

\section{(cc) $\mathrm{Br}$}

Este trabalho está licenciado com uma Licença Creative Commons - Atribuição 4.0 Internacional. 